\title{
Assessment of Quality of Life and Hazard Issues Involving Worth of Life in Adolescent Idiopathic Scoliosis
}

\author{
Irena Kola ${ }^{1}$, Sander Kola ${ }^{2}$, Gjovalin Valca ${ }^{3}$, Enkelejda Shkurti ${ }^{4}$, Diamant Shtiza ${ }^{5}$ \\ ${ }^{1,4}$ Faculty of technical Medical Sciences, University of Medicine, Tirana, Albania \\ ${ }^{3}$ Rreshen Hospital, Pediatrics department, Albania \\ ${ }^{2,5}$ University Hospital Centre: "Mother Theresa”, Tirana, Albania
}

\begin{abstract}
Background: Adolescent idiopathic scoliosis (AIS) is a frequent illness guiding to spinal distortion in children ages 10 and above. With the progress in the survey of health-related quality of life (HRQoL), bigger awareness has been offered to the quality of life (QoL) of subjects with AIS and their insight of distortion as opposed to just focusing on increasing the rate of surgical modification. Aim/Methods: This review affords a summary of the techniques of assessing HRQoL and it examines numerous major issues influencing QoL, like harshness of illness, technique of healing, sex, and social setting, based on preceding surveys of patients with AIS. Results: Support treatment is an essential outline of traditional treatment for patients with AIS, and this therapy can considerably decrease the harshness and the progress of AIS. Various investigators consider that patients with AIS who experience brace therapy may sense timid and have interior stress as an outcome of lifestyle or studying. Consequently, the mental wellbeing of patients with AIS should be assessed and checked to decrease the harmful psychosomatic results of brace treatment. Conclusion: The researchers consider that radiological surveys should no longer be acquired as the single sign of postoperative remedial appraisal and expect to construct a new appraisal scheme with estimation of QoL for patients with AIS.
\end{abstract}

Keywords: Adolescent idiopathic scoliosis, quality of life, assessment, questionnaire, hazard issues

\section{Introduction}

Adolescent idiopathic scoliosis (AIS) is described by the Scoliosis Research Society (SRS) as an unidentified spinal distortion with a coronal Cobb angle $>10$ grades arising in a kid over the age of 10 whose skeleton is still extending. AIS happen commonly in adolescents. The rate at which AIS is assessed has significantly developed with the formulation of the assumption of three dimensional scoliosis adjustment and quick progress in interior fixation, such as multi-level pedicle rotate fixation. Alterations in healthcare patterns and stable progress in investigations into health-linked value of life (HRQoL) have led to the apprehension that superior awareness should be given to the quality of life (QoL) of patients with AIS and their awareness of malformation in preference to just focusing on advancing the rate of surgical adjustment. While scoliosis is away from life-menacing, public, family, and surgery-connected aspects might guide patients to build up mental confusions (Tones M, Moss N, Polly DW Jr 2006) or even challenge suicide (Payne WK $3^{\text {rd }}$ et al 1997). So as to improve patient QoL and contentment with cure, awareness must be given to investigate on QoL in patients with AIS.

\section{Methodology}

HRQoL is correlated with health condition and is a person's level contentment or fulfillment with individual life affairs in the expression of illness, accident or harm or medical management. HRQoL reveals a patient's individual appraisal of his or her Quality. Measurements of health status, occupation, ache, and contentment can be assessed using complete scales and questionnaires that evaluate universal health or the situation of a specific illness. Ordinary HRQoL scales are split into two groups, universal devices to estimate HRQoL and particular tools to appraise HRQoL.

Short Form-36 Health Survey (SF- 36) is the questionnaire most frequently used to appraise common health. Further tools comprise the Pediatric Outcomes Data Collection Instrument (PODCI) and the Child Health Questionnaire (CHQ). The SF-36 involves the 8 features of physiological performance, corporal pain (BP) etc.

SF-36 can be applied to appraise the QoL of patients with diverse illnesses (Ware JE. Sherbourne CD 1992). While the SF-36 is usually utilized in medical practice, it is a universal device to assess HRQoL and it not precise to scoliosis. Other disadvantages are issues such as recurring matters and the long time required to manage the survey. Furthermore, estimation of self-esteem, which is of meticulous impact in Exact devices to appraise HRQoL are specially intended for a precise illness such as spinal distortion. Tools to appraise QoL in subjects with scoliosis comprise the Scoliosis Research Society Outcomes Instruments (SRS-22 and SRS24), the Quality of Life Profile for Spinal Deformities (QLPSD), the Spinal Appearance Questionnaire (SAQ (Climent JM at 1997\& Feise RJ 2005\& Asher M).

Haher et al. (1995) generated the easy and convenient SRS24 HRQoL survey for subjects with scoliosis in 1999 and they asserted that a kid's HRQoL and individual fulfillment should as well be appraised as ingredient of the estimation after operation for scoliosis. The SRS-24 is separated into two fractions. The primary element comprises evaluation of ache, performance, self-esteem, and movement, and this section can be utilized to assess any individual with 


\section{International Journal of Science and Research (IJSR) \\ ISSN (Online): 2319-7064}

Index Copernicus Value (2013): 6.14 | Impact Factor (2014): 5.611

scoliosis. The subsequent fraction incorporates postoperative self-esteem, performance, and contentment with healing. This part can simply be applied to appraise patients after operation for scoliosis.

The SRS-24 has numerous benefits such as being dissimilar and succinct and having an elevated answer rate. Nevertheless, the next fraction of the range is limited to subject assessment after operation. In 2000 and 2003, Asher established a personalized SRS and SRS-22 survey comprising five phases of practical condition, self-esteem, ache, emotional position, and accomplishment with cure. This adapted range is moreover more precise in various aspects than the SF-36. Additionally, the SRS-22 can be used to assess QoL in subjects after operation for scoliosis plus QoL in patients getting traditional cure of scoliosis. The SRS-22 is the world's mainly applied scale to appraise the QoL of patients with scoliosis.

Sanders et al. (2007) generated an original HRQoL questionnaire identified as SAQ in 2007. The SAQ merges identical illustrations with a survey to appraise how patients and their families personally sense about a spinal distortion. Sanders also found that the SAQ was more susceptible and consistent in distinguishing a progress in QoL after operation than the SRS-22. Wei et al. (2011) produced a Chinese easy version of the SAQ that was ethnically tailored in agreement with global instructions. They revealed its good reliability and validity in estimating how patients with AIS in China rated their look.

\section{Results}

With the expansion of the Bio-Psycho-Social representation of human performance (Hemsworth PHet al 2002 \& Kraaij V 2010) and constant alterations to suitable questionnaires, larger significance has been joined to issues that influence a patient's QoL. A survey by Payne et al. (1992) specified that the occurrence of a spinal distortion was a hazard issue for psychological dejection regardless of of the treatment the patient acquired. Adolescence is a susceptible phase of individual and psychosomatic progress, so numerous features similar to a deformation and physical uneasiness can involve the QoL of patients with AIS.

Patients with AIS are frequently observed for an deformity like inaccurate body position or left-right irregularity of the shoulders. While adolescence is a serious stage of psychological progress, the malformation caused by scoliosis may put a certain level of social and psychosomatic stress on patients, and a more harsh distortion will be the reason of larger mental pressure. AIS is a multifaceted threedimensional distortion. Spinal distortion in any aircraft can influence a patient's outcomes on an HRQoL questionnaire. For postoperative patients with AIS, the Cobb approach of the instrumented thoracic arch is the major issue persuadeing QoL. A survey by Helenius et al. (Helenius I \& Remes V 2002) inspected 98 successive patients who experienced surgery with a Harrington disruption rod and posterior spondylodesis. They found that the magnitude of thoracic curvature as assessed during follow-up an average of 21 years later advances comprise psychosomatic testing to evaluate patient individuality categories prior to stimulating and devising tailored cure diagrams for individual patients to offer an enhanced QoL.

The result of the operation on a patient's common and psychosomatic performance has obtained less awareness in the literature than the outcomes of support treatment on that performance. Operation is a main confront for patients with AIS because of problems such as ache and expressive sorrow through hospitalization, concerns regarding surgical problems, and the interference to one's social life through post-surgical recuperation (LaMontagne LL et al 2004). A composite illness, AIS is not voluntarily cured with operation and patients with AIS as well have an elevated hazard of afflicting emotional disease, chiefly as an outcome of features like preoperative trait concern and a small stage of cognitive growth. Consequently, close awareness must be given to a patient's psychosomatic situation and mental intrusion must be offered when required besides correcting scoliosis.

Sex is an issue that involves the psychology of subjects with AIS. Payne et al. (1997) applied the Adolescent Health Survey (AHS) to examine 685 subjects with AIS, 274 males and 435 females varying in age from 13-17years. The AHS is a complete appraisal of health condition that challenges to establish all remedial, social, and relatives conditions that might have a collision on the health position of youngsters. The survey's outcomes pointed out that scoliosis was an autonomous risk aspect for more recurrent suicidal notions, more distress about irregular body growth, and a superior concern about friends relations. Male youngsters with scoliosis were $60 \%$ more prone to believe they were malnourished whereas female youngsters with scoliosis were $48 \%$ more likely to have suicidal notions than their friends. This involves that the collision of scoliosis and sex distinctions in patients may be superior than formerly thought.

Albania is a developing nation with unequally developing local economies. There are significant discrepancies in living circumstances, profits, and medical structures in urban and countryside regions. Matched up to rural regions, urban ones allocate a more open existence with elevated earnings and a enhanced health insurance structure. The social issues are certain to influence the appraisal of a patient's QoL. These differences that will be exposed in SRS-22 were inversely associated with scores for aesthetic features on the SRS-24. A survey by Watanabe et al. (2007) discovered that universal self-esteem was inversely linked to the Cobb angle and the rotational movement angle of the thoracic curve and that self-esteem after operation was linked with the extent of adjustment of the thoracic Cobb angle. These outcomes point out that the Cobb angle of the thoracic curve and radiographic strictures for the assessment of scoliosis in the axial plane significantly influence patient results, and mainly how patients with AIS measure recognize their look. A survey by Shang et al. (2009) achieved 52 patients with AIS applying the Symptom Checklist 90 (SCL-90), Self rating Depressive Scale (SDS), and Self-rating Anxiety Scale (SAS). They evaluated those results to those of 50 healthy young volunteers of the same age. They investigated that patients with harsh AIS were more likely to have

\section{Volume 4 Issue 11, November 2015}




\section{International Journal of Science and Research (IJSR) \\ ISSN (Online): 2319-7064 \\ Index Copernicus Value (2013): 6.14 | Impact Factor (2014): 5.611}

psychosomatic problems, therefore influencing their QoL, than patients with medium or gentle AIS

Support treatment is an essential outline of traditional treatment for patients with AIS, and this therapy can considerably decrease the harshness and the progress of AIS (Zhu ZZ, Qiu Y 2004\& Lonstein JE et al.2015). Nevertheless, stimulating is probable to cause undesirable psychosomatic stress that influences QoL. A survey by Climent et al. (1999) applied QLPSD to evaluate the outcome of diverse sorts of braces on QoL, and they investigated that patients cured with a Milwaukee brace achieved considerably higher than patients that a Boston brace, particularly in terms of psycho-social performance. This which means that the Milwaukee support has a bigger collision on QoL. A survey by Maruyama et al. (2011) applied the SRS-22 to assess the QoL of patient scured with a Milwaukee brace and they accomplished the same end. Matsunaga et al. (2004) applied the Maudsley Personality Inventory to assess 152 adolescent girls with idiopathic scoliosis to evaluate alterations in behavior subsequent to brace treatment Of the 129 patients rated as normal previous to the beginning of therapy, 102 were rated as atypical when checked 1 month following the initiation of treatment. Subsequent to psychosomatic interference, 52 patients were lastly rated as anomalous, which implies that psychosomatic tough combined with psychological cure may decrease the harmful psychological outcomes of brace treatment and assist tailored bracing. Various investigators consider that patients with AIS who experience brace therapy may sense timid and have interior stress as an outcome of lifestyle or studying. Consequently, the mental wellbeing of patients with AIS should be assessed and checked to decrease the harmful psychosomatic results of brace treatment. A survey by Wang et al. (2008) applied the SRS-22 to revise the county issues that influenced patient QoL, and they investigated that urban patients had extensively elevated scores in approval with administration of their illness and inferior scores in self-esteem than did country subjects. This point outs that dissimilarities among urban and rural regions influence the assessment of QoL.

For subjects with AIS, the family setting is furthermore an issue that influences QoL. Kahanovitz and Weiser (2014) examined 72 adolescent girls with scoliosis ages 11- 18 years, and they investigated that subjects from single parent families had a minor QoL and that the mother's approach towards her kid's disease had a very positive consequence on a kid's approaches in the direction of healing, hence improving his or her QoL. Different grown-ups, the majority of kids fail to fulfill the cure and recuperation procedure as a result of their detailed physiological features. The psychosomatic situation of parents straight influences their kids, who are probably to implement the performance and attitudes of their parents. Consequently, the parent kid connection should be highlighted when caring for teenager subjects.

\section{Conclusion}

In summary, the QoL of subjects with AIS can be straight or ultimately influenced by aspects such as illness, healing, individual qualities, and social conditions. This information is being recognized by spinal doctors. Complete and helpful questionnaires or degrees for the precise illness (scoliosis, in this case) must be utilized to summarize on a patient's QoL and untimely mental interference must be offered when required. Radiological surveys should not provide the solitary technique of postoperative assessment in patients with AIS and appraisal should comprise estimation of QoL.

\section{References}

[1] Tones M, Moss N, Polly DW Jr 2006. A review of quality of life and psychosocial issues in scoliosis. Spine ; 31:3027-3038.

[2] Payne WK 3rd, Ogilvie JW, Resnick MD, Kane RL, Transfeldt EE, Blum RW 1997. Does scoliosis have a psychological impact and does gender make a difference? Spine; 22:1380-1384.

[3] Ware JE. Sherbourne CD 1992. The MOS 36-item short-form health survey (SF-36). Conceptual framework and item selection. Med Care. 30:473-483.

[4] Climent JM, Reig A, Sánchez J, Roda C 1995. Construction and validation of a specific quality of life instrument for adolescents with spine deformities. Spine; 20:2006-2011.

[5] Feise RJ, Donaldson S, Crowther ER, Menke JM, Wright JG 2005. Construction and validation of the scoliosis quality of life index in adolescent idiopathic scoliosis. Spine ; 30:1310-1315.

[6] Asher M, Min Lai S, Burton D, Manna B 2003. The reliability and concurrent validity of the scoliosis research society-22 patient questionnaire for idiopathic scoliosis. Spine; 28:63-69.

[7] Asher M, Min Lai S, Burton D, Manna B 2003. Scoliosis research society-22 patient questionnaire: Responsiveness to change associated with surgical treatment. Spine ; 28:70-73.

[8] Haher TR, Merola A, Zipnick RI, Gorup J, Mannor D, Orchowski J 1995.Meta-analysis of surgical outcome in adolescent idiopathic scoliosis: A 35-year English literature review of 11,000 patients. Spine; 20:15751584.

[9] Li M, Wang CF, Gu SX, He SS, Zhu XD, Zhao YC, Zhang JT 2009. Adapted simplified Chinese (mainland) version of Scoliosis Research Society-22 questionnaire. Spine; 34:1321-1324.

[10] Li M, Wang CF, Gu SX, He SS, Zhu XD 2008. Reliability and validity of adapted simplified Chinese version of the Scoliosis Research Society-22 patient questionnaire. Chinese Journal of Spine and Spinal Cord; 3:212- 217. (in Chinese)

[11] Sanders JO, Harrast JJ, Kuklo TR, et al 2007. The spinal appearance questionnaire: Results of reliability, validity, and responsiveness testing in patients with idiopathic scoliosis. Spine; 32:2719-2722.

[12] Wei XZ, Li M, Chen JY, Wang CF, Zhang GY, Wu b, Liu XS, Zhang W, Chen ZQ, L M 2011. Primary application of the simplified Chinese version of spinal appearance questionnaire in appearance evaluation of adolescent idiopathic scoliosis. Journal of Spinal Surgery; 6:342-344

[13] Hemsworth PH, Coleman GJ, Barnett JL, Borg S, Dowling S 2002. The effects of cognitive behavioral intervention on the attitude and behavior of

\section{Volume 4 Issue 11, November 2015}




\section{International Journal of Science and Research (IJSR) \\ ISSN (Online): 2319-7064}

Index Copernicus Value (2013): 6.14 | Impact Factor (2014): 5.611

stockpersons and the behavior and productivity of commercial dairy cows. J Anim Sci ; 80:68-78.

[14] Kraaij V, van Emmerik A, Garnefski N, Schroevers MJ, Lo-Fo-Wong D, van Empelen P, Dusseldorp E, Witlox R, Maes S 2010. Effects of a cognitive behavioral selfhelp program and a computerized structured writing intervention on depressed mood for HIV-infected people: A pilot randomized controlled trial. Patient EducCouns ; 80:200-204.

[15] Helenius I, Remes V, Yrjönen T, Ylikoski M, Schlenzka D, Helenius M, Poussa M. 2002 Comparison of long-term functional and radiologic outcomes after Harrington instrumentation and spondylodesis in adolescent idiopathic scoliosis: A review of 78 patients. Spine; 27:176-180.

[16] Watanabe K, Hasegawa K, Hirano T, Uchiyama S, Endo N 2007. Evaluation of postoperative residual spinal deformity and patient outcome in idiopathic scoliosis patients in Japan using the scoliosis research society outcomes instrument. Spine ; 32:550-554.

[17] Shang GL, Fan X, Miao DM, Tao HR, Luo ZJ 2009. Analysis of psychological characteristics in adolescent idiopathic scoliosis. Chinese Journal of Spine and Spinal Cord ; 3:204-207.

[18] Zhu ZZ, Qiu Y, Wang B, Yu Y, Li WG, Zhu LH 2004; Clinical outcomes of bracing in adolescent idiopathic scoliosis. Chinese Journal of Orthopaedics. 24:276280.

[19] Lonstein JE, Winter RB 1994. The Milwaukee brace for the www.irdrjournal.com 16 Intractable \& Rare Diseases Research. 2015; 4(1):12-16. treatment of adolescent idiopathic scoliosis: A review of one thousand and twenty patients. J Bone Joint Surg Am ; 76:1207-1221.

[20] Climent JM, Sánchez J 1999. Impact of the type of brace on the quality of life of adolescents with spine deformities. Spine ; 24:1903-1908.

[21] Maruyama T, Takeshita K, Kitagawa T, Nakao Y 2011.Milwaukee brace. Physiother Theory Pract; 27:43- 46 .

[22] Matsunaga S, Hayashi K, Naruo T, Nozoe S, Komiya S 2005. Psychologic management of brace therapy for patients with idiopathic scoliosis. Spine ; 30:547-550.

[23] LaMontagne LL, Hepworth JT, Cohen F, Salisbury MH 2004. Adolescent scoliosis: Effects of corrective surgery, cognitive-behavioral intervention, and age on activity outcomes. Appl Nurs Res ; 17:168-177.

[24] Wang CF, Li M, Gu SX 2008. Is living domain affect patient's outcome in postoperative adolescent idiopathic scoliosis patients using the scoliosis research society outcomes instrument? Chinese Journal of Spine and Spinal Cord; 11:820-823.

[25] Kahanovitz N. Weiser S. 2014. The psychological impact of idiopathic scoliosis on the adolescent female. A preliminary multi-center study. Spine. 1989; 14:483485. (Received December 10; 